\section{Ecturs}

'Department of Thoracic Surgery, Birmingham Heartlands Hospital, Birmingham, UK Institute of Cardiovascular Sciences, University of Birmingham, Birmingham, UK ${ }^{3}$ School of Medicine, University of Birmingham, Birmingham, UK ${ }^{4}$ Department of Histopathology, Birmingham Heartlands Hospital, Birmingham, UK

\section{Correspondence to}

Maninder S Kalkat, Department of Thoracic Surgery, Birmingham Heartlands Hospital, Bordesley Green East, Birmingham B9 5SS, UK;

maninder.kalkat@ heartofengland.nhs.uk

Received 23 September 2016 Accepted 7 October 2016

Published Online First

31 October 2016

\title{
Cholesterol granuloma of the anterior mediastinum
}

\author{
Nigel E Drury, ${ }^{1,2}$ Danielle NE Smith, ${ }^{3}$ Lucy M Phillips, ${ }^{3}$ Simon E Trotter, ${ }^{4}$ \\ Maninder S Kalkat ${ }^{1}$
}

A 74-year-old man presented with dyspnoea and reduced exercise tolerance (Medical Research Council grade 4). He was a current smoker with a history of paroxysmal atrial fibrillation and COPD for which he was on bronchodilators. There was no history of previous chest trauma or surgery and physical examination was unremarkable. CT thorax showed a $3.2 \mathrm{~cm}$ mass in the anterior mediastinum with two foci of coarse calcification (figure 1A). Positron emission tomography (PET)/CT demonstrated significant uptake (maximum standardised uptake ratio of 13.7) in the mass with a central area of inactivity (figure 1B); there was no significant activity elsewhere. His gas transfer was reduced at $63 \%$ predicted.

The differential diagnosis included a thymic tumour or pathological lymph nodes, and excision biopsy was recommended to establish a histological diagnosis. A right video-assisted thoracic surgery (VATS) total thymectomy was performed and the macroscopic appearance was consistent with a thymic tumour (figure 2A). However, on histological examination, the mass comprised two separate well-circumscribed lobules composed exclusively of cholesterol crystal inclusions supported by a fibrous background matrix with foreign body type giant cells and areas of dystrophic calcification, consistent with a mediastinal cholesterol granuloma (figure 2B); there was no evidence of malignancy.

Cholesterol granuloma is an uncommon, benign pathological entity that is thought to be initiated by localised trauma or an inflammatory response predisposing to microscopic haemorrhage. Haemolysis liberates cholesterol from the cell membranes of degenerating erythrocytes to form crystals, which stimulate a foreign body type giant cell reaction, leading to chronic inflammation and granuloma formation. ${ }^{1}$ An alternative pathogenesis involving inflammation in a benign cystic tumour has also been proposed. ${ }^{2}$

Cholesterol granulomas, occurring most frequently in the petrous apex of the temporal bone adjacent to the middle ear, are associated with chronic infections and if left untreated, may cause bony erosion and cranial nerve injury. They have also been identified in the kidney, breast, peritoneum, parotid, testis, lung, liver and spleen with only a few cases reported in the mediastinum, including as an incidental finding at median sternotomy for cardiac surgery. ${ }^{1}$ There are no reports of a mass effect or erosion of structures in the mediastinum.

The preoperative diagnosis of a mediastinal cholesterol granuloma may be challenging due to its rarity and radiological appearances resembling more common, sinister lesions. With a high uptake, fluorodeoxyglucose-PET proved unhelpful in differentiating it from a malignant tumour. Magnetic resonance may be valuable as it has been shown to have markedly low signal on T1- and T2-weighted imaging due to abundant hyalinised collagen fibres; ${ }^{3}$ however this is not performed routinely in the evaluation of an anterior mediastinal mass. Indeed, as complete surgical resection is the standard of care for suspected thymic neoplasm, excision should be performed to confirm the diagnosis.

In conclusion, cholesterol granuloma is an extremely rare cause of an anterior mediastinal mass that may masquerade as a more sinister pathology or be found incidentally during cardiac surgery. While
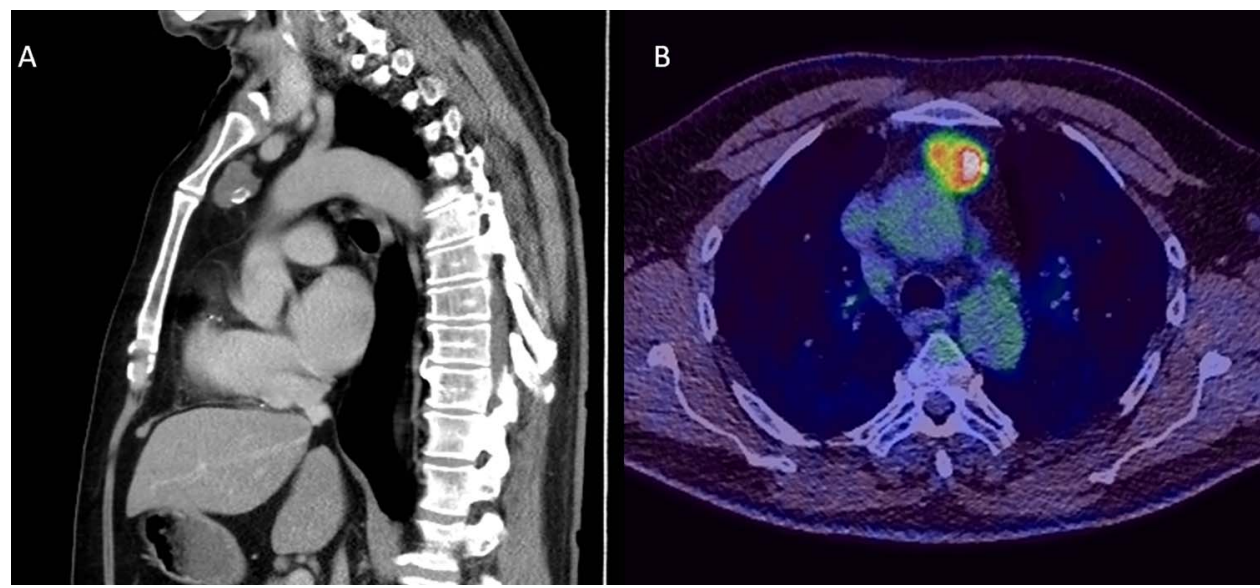

Figure 1 CT sagittal plane showing a $3.2 \mathrm{~cm}$ lesion in the anterior mediastinum with areas of focal calcification (A) and positron emission tomography/CT demonstrating significant metabolic activity (maximum standardised uptake value of 13.7) in this mass (B). 


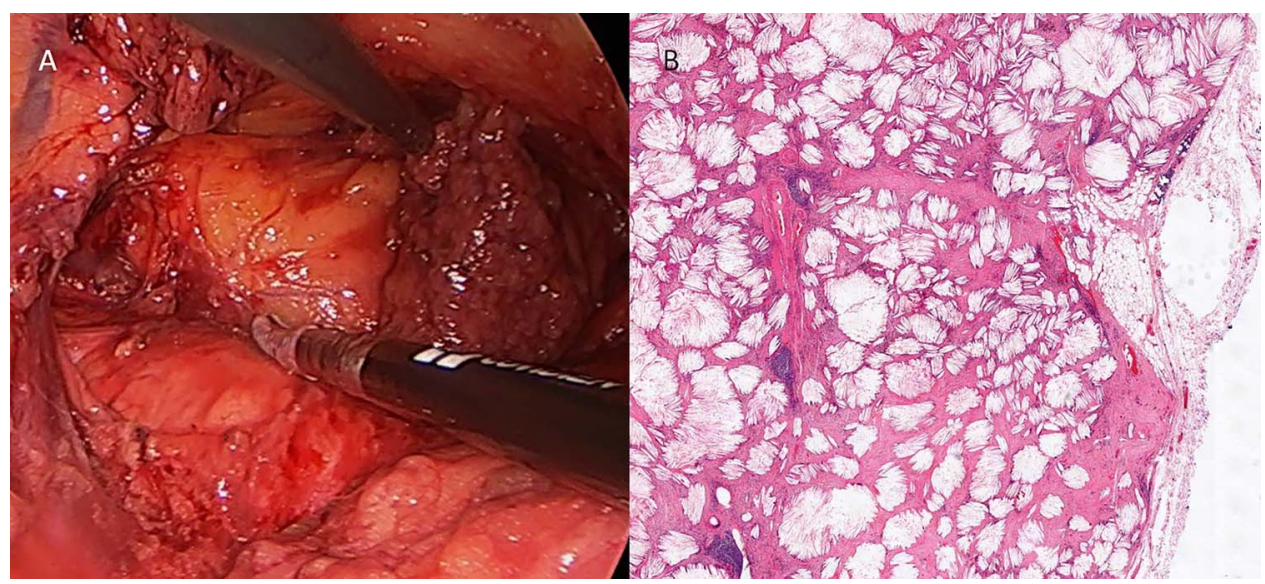

Figure 2 At video-assisted thoracic surgery, the anterior mediastinal mass was found to be adherent to the pericardial reflection over the ascending aorta with which it was removed (A) and on microscopy, low power view showed a well-defined lesion comprising numerous cholesterol inclusions supported by a fibrous background matrix (H\&E) (B).

its histological appearance is characteristic, the radiological diagnosis may prove more challenging and therefore surgical excision is indicated to exclude a thymic or other malignancy.

Contributors All authors were involved in composing the manuscript. Competing interests None.

Patient consent Obtained.

Provenance and peer review Not commissioned; externally peer reviewed.

\section{REFERENCES}

1 Krishnan TR, Sinha SK, Kejriwal NK. A rare case of cholesterol granuloma in the anterior mediastinum. Heart Lung Circ 2013;22:303-4.

2 Weissferdt A, Kalhor N, Moran C. Primary thymic cholesteroloma: a clinicopathological correlation of four cases of an unusual benign lesion. Virchows Arch 2015;467:609-11.

3 Fujimoto K, Takamori S, Yano H, et al. Focal cholesterol granuloma in the anterior mediastinum: [18F]-fluoro-2-deoxy-D-glucose-positron emission tomography and magnetic resonance imaging findings. J Thorac Oncol 2007:2:1054-6. 